\title{
Surgery for Cancer in the Wake of Second and Future Waves! Challenges and Opportunities in the Indian Context
}

\author{
Narendra Hulikal ${ }^{1}\left[\right.$ D Singaram Nagesh Kumar $^{1} \cdot$ Kodaganuru S. Gopinath $^{2,3}$
}

Received: 23 May 2021 / Accepted: 19 July 2021 / Published online: 30 July 2021

(C) Indian Association of Surgical Oncology 2021

\begin{abstract}
The pandemic situation created by newly discovered severe acute respiratory disease corona virus 2019 has thrown up many challenges to surgical fraternity across world. All surgeons particularly cancer surgeons are facing dilemmas not only in managing patients but also in appropriate personal as well as employee's health protection as well. Since the pandemic is not expected to recede unless it affects a considerable proportion of population or up to $60 \%$ of population is vaccinated, we need to be aware of prevailing best practices. This is important in ensuring continuity of care for cancer patients as well as to limit the consequences of one getting infected with the COVID-19. This review article focuses on the current challenges and the ways to mitigate them.
\end{abstract}

Keywords Severe acute respiratory syndrome corona-2 virus disease 2019 · COVID-19 - Cancer surgery during covid pandemic

The pandemic situation created by newly discovered severe acute respiratory syndrome corona-2 virus (SARS Cov-2) disease 2019 (COVID-19) has severely impacted all spheres of life including medical care. The first confirmed case of COVID-19 in India was reported from Kerala in late January last year [1], followed soon by first wave of pandemic, reaching nearly 100,000 infections per day at peak, receding by late September after claiming 150,000 lives [2]. Several measures including months of lockdown and gradual opening may have succeeded in limiting the final numbers, though the economic consequences were severe. The current wave which started in late March is proving to be more dangerous as the nation seems to be underprepared. All the epidemiologists are of the view that the pandemic will not end unless a substantial population is vaccinated, or the population acquires a reasonable degree of herd immunity through

Narendra Hulikal

drnarendrah@yhoo.co.in

1 Department of Surgical Oncology, Sri Venkateswara Institute of Medical Sciences, Tirupati, Andhra, Pradesh 517507, India

2 HCG-Bangalore Institute of Oncology, Bangalore, India

3 Surgery \& Oncology, Sri Devaraj Urs Medical College, No. 20, Srinivasa Behind, Kolar, Balaji Kalyana Mantapam, BSK III Stage, Bangalore 560085, India natural infection. In this regard planning to carry on with the routine medical care for non-COVID-19 diseases especially cancer becomes important. The cocktail of COVID in cancer patient throws up many challenges to a clinician.

\section{Routine SARS Cov-2 Testing and COVID-19 Care in a Cancer Patient!}

Incidence of COVID-19 in cancer patients is variable, most data suggesting 1-8\% with highest risk among hematologic and lung cancers [3, 4]. Centre for Disease Control (CDC), USA, considers cancer as one of established risk factor for severe COVID-19 but data is weak to say the risk is same across all cancers. Patients with advanced age, progressive disease and comorbidities are at increased risk of death [4]. Though international guidelines vary regarding routine COVID testing, generally testing is advisable given the community prevalence of the infection in India $(>10 \%$ PCR positivity in a community), for time sensitive surgeries and procedures involving aerosol generation. American Society of Clinical Oncology (ASCO) recommends testing only in the presence of COVID symptoms [5], whereas Infectious Disease Society of America (IDSA) recommends routine testing for patients undergoing immunosuppressive procedures like chemotherapy [6]. As antigen tests carry 
low sensitivity, nucleic acid amplification tests are preferred using reverse transcriptase polymerase chain reaction (RTPCR). Further, repeat testing is not needed if infected in the past 3 months as there is less chance of active infection, even if tested positive.

The actual management of COVID-19 infection will not differ from non-cancer patients and available data suggest that patient with cancer who develop COVID-19 are not necessarily at increased risk of hypercoagulable state [7]. The need for ventilatory support in any cancer patient with advanced stages or comorbidity for severe COVID infection carries a grim prognosis and needs a proactive discussion about goals of care with patient relatives [8, 9].

\section{Cancer Care in a COVID-Positive Patient!}

Multidisciplinary care of cancer when a patient tests positive poses unique challenge as it involves ethical, consistent, and transparent decision-making process involving all frontline stakeholders [10]. As of September 2020, ASCO guidelines recommended that until more definitive information is available, decision about withholding anti-cancer treatment should be based on clinical risk benefit analysis that considers the risk of interrupting cancer treatment versus yet poorly defined risk of adverse COVID-19 outcomes in patient receiving active cancer treatment. Further, when treatment decisions are made, such patients should be treated in specific areas with adequate COVID care facilities and dedicated staff [11]. The risk of perioperative morbidity and mortality may be increased in patients with COVID, and decision to perform urgent or emergent surgery must balance this risk against the risk of delaying or avoiding the planned procedure. Procedures that are not time sensitive should not be performed in symptomatic COVID patients, who are suspected of having COVID-19 or who are likely to be still infected after having had COVID-19. One study showed that patients who underwent surgery within 7 weeks after diagnosis of COVID had higher 30-day mortality compared to those who underwent surgery after 7 weeks with odd's ratio of 4:1.5. Even after 7 weeks, persistence of symptoms was associated with higher mortality and hence wherever possible, surgery can be delayed beyond 7 weeks [12]. According to ASCO for anti-cancer treatment to start in immunocompromised patients, one has to wait for 20 days and 10 days for non-immunocompromised patients with two negative COVID tests, more than $24 \mathrm{~h}$ apart along with absence of symptoms like cough and fever (in the absence of any antipyretic medication). Persistent viral shedding after recovery has several implications such as infectivity, continued infection control precautions, delay in investigations, and treatment. For most patients, prolonged or recurrent viral RNA detection does not necessarily indicate prolonged infection; isolation of infectious virus from upper respiratory tract specimen more than 10 days after onset of illness has rarely been documented in an asymptomatic patient with non-severe infection. It is recommended not to delay cancer procedures or surgeries if patient is persistently COVID19-positive based on cycle threshold as measured by RTPCR [13]. Though It is established well that patient with active cancer are at increased risk for severe COVID disease and mortality, whether cancer survivors who have completed treatment are at increased risk for COVID-19 or its complications is still unclear. Other emerging issue at the time of writing this article is challenge of managing post-COVID complications including mucor mycosis in immunocompromised patients including cancer patients.

\section{Cancer Care in Non-COVID Patients in the COVID Times!}

Coming to cancer care for a patient who has no COVID infection, the world is not the same anymore. Oncologists must balance the risk of delaying cancer treatment versus risk of SARS Cov-2 exposure and its complications while navigating with limited health care resources. In order to provide safe care, one can follow general guidelines given by $\mathrm{CDC}$ and ASCO and several associations including Indian Association of Surgical Oncology [14]. Generally, ASCO recommends segregating patients based on their COVID test status to avoid contact with symptomatic COVID patients; mandatory universal wearing of face mask, following a strict hand washing protocol, to cancel all routine screening and follow-up visits and extending the time between follow-up visits. Pre-visit COVID screening via telephone calls or digital platforms 48 to $72 \mathrm{~h}$ before scheduled appointment for any history of COVID exposure in the past 14 days or any COVID symptoms in the preceding 10 days is highly recommended [15]. Majority of routine surveillance consultations can be done with teleconsultations following the guidelines issued by erstwhile Medical council of India [16]. Screening clinics should be developed to allow for patient with symptoms to be evaluated. Routine cancer screening and diagnostic clinics should be based on the status of COVID-19 in that community. American Society of Clinical Oncology recommends postponing screening clinics to decrease the burden on health care system and to preserve the resources. Screening clinic can be restarted once the infection rate comes down in the community.

Though at present, there is no evidence that COVID-19 interferes with or influences the diagnosis and staging of cancer, it is important to limit the number of staging procedures to decrease number of visits. There is an evolving situation that COVID-19 vaccination can cause axillary and supraclavicular adenopathy which may interfere with 
cancer staging and one needs to be cautious in reporting and interpreting these findings. A screening mammogram may be planned either before first dose of vaccination or 4 to 6 weeks following the second dose [17]. Though a delay in cancer treatment can result in adverse oncologic outcomes depending on cancer type and stage, one must weigh the risk and benefit given the current pandemic situation in each community. One study revealed that a delay of 4 weeks was associated with increased mortality across surgery, radiotherapy, and chemotherapy for bladder, breast, colon, head, and neck including nasopharyngeal, cervical, and non-small cell lung cancers [18]. Some of the online tools such as oncCovid can help clinicians to estimate the risk of delaying treatment for individual patient. It can give a reasonable estimate of 5-year mortality, cancer-specific mortality, hazard ratios of treatment delays for various intervals and risk of developing and dying from COVID-19 over 6 months period [19].

Since many cancer surgeries including some palliative procedures are time sensitive need special mention. Treatment of many cancers such as brain tumors, cancers of breast, colon, stomach, pancreas, liver, bladder, kidney, and lung cannot wait for 2 to 3 months as patients would be deprived of significant surgical benefit [20]. In some like breast, prostate, and rectal cancer, neoadjuvant therapy may be used as a means of delaying surgery. In rectal cancer, total neoadjuvant therapy can be safely followed neoadjuvant chemotherapy or hormonal treatment in breast cancer and neoadjuvant hormonal treatment in risk prostate cancer can be employed [21]. Similarly, radiotherapy can be used for many of oral cancers and cancers of upper aero digestive tract including esophagus. When such decisions are made risk of tumour progression with delay in definitive surgery should be weighed against the potential added burden on hospital resources, case complexity and risk of COVID-19 exposure. However, neoadjuvant therapy that is immunosuppressive or requires frequent clinic visit has potential incremental risk. The rationale for delaying surgeries is conservation of resources, limitation of viral spread, and minimizing postoperative infection and mortality [22]. Non-emergent, non-time-sensitive surgeries can be resumed if there is downward trend of community infection rate; resources are available, where testing of patient and staffs is adequate. One of the useful model could be on the lines of American College of Surgeons who endorse use of cross discipline surgical prioritization system called "Medically-Necessary, Time-Sensitive Procedures" (MeNST) score which is calculated for each case over 21 distinct items [23]. One more approach could be conceptual approach to decision-making regarding timing cancer treatment which attempts to balance the risk of progression with risk of significant morbidity from COVID-19. In this approach, owing to low risk of progression, it may be safer to delay treatment for more than
3 months for certain cancers like non-melanoma skin cancer or hormone receptor positive her- 2 negative early breast cancer regardless of age. Some of the cancers with intermediate risk of progression, a delay of approximately 3 months, may be acceptable in individuals less than 50 years old and older with intermediate to high-risk prostate cancer (can be on hormonal treatment), low-risk melanoma, and colon cancer without impending obstruction. For certain cancers with high risk of progression, ideally there should be no delay in treatment for individuals under the age of 70 . This category includes patients with more than $2 \mathrm{~cm}$ lung mass, colon cancer with imminent obstruction, type 2 endometrial cancer, cervical cancer, pancreatic, ovary, liver malignancy, high-risk non-muscle invasive, and muscle invasive bladder cancer who are not eligible for chemotherapy, early testicular cancer, invasive penile cancer, and patients who completed preoperative radiotherapy for locally advanced rectal cancer [24-26]. Again, a note should be made that most of these frame works are expert opinion than evidence-based recommendations. Data from National Cancer Database (NCDB) reveals that for common solid tumors, such as cancers of breast, prostate, lung, and colon, shorter time to initiation of treatment is associated with lower mortality. In addition to potentially altering treatment protocols, other actions suggested to increase the safety of managing oncologic care during pandemic include screening for COVID-19, limiting exposure to contacts on treatment, minimizing non-essential visits, restricting visitors, and increased engagement in telemedicine. COVID-19 pandemic has put telemedicine into limelight because it is accessed by people directly from home, reduces viral transmission, and safeguards patient and staff. Some of the challenges include data privacy, medical liability access to devises, difficulty in understanding how to use telemedicine equipment, incompleteness of physical examination, decreased ability to participate in clinical trials, and hampered resident training and difficulties in coordinating care. Accumulating data support mostly favorable outcomes. Appropriate and agile implementation of a virtual care delivery system successfully enabled the continuity of outpatient cancer care delivery during pandemic [27, 28].

\section{Impact of COVID-19 on Resident Training and Clinical Trials}

The impact of COVID-19 on surgical training of oncological residents is many folds. In many of the public sector hospitals, workforce has been diverted from their field of specialization to care of COVID-19 patients, thereby leading to compromises in speciality training. Multiple lockdowns have also resulted in reduction in patient volume leading to non-uniform effects across hospitals. Even the mode of conduct of exams has been modified to suit the new norm. At 
the time of writing this article, the national board of examinations has extended the period of training by 3 months and national eligibility entrance test for admission to first year broad and super speciality courses have been indefinitely delayed [29, 30]. All these would have certain impact on over all cancer care, the magnitude of which is unknown at this time. The role of clinical trials in transforming cancer care is beyond doubt. The conduct of clinical trials is often seamlessly integrated into routine oncology care offering eligible patients additional treatment options. The COVID19 pandemic has become the major barrier for new patient recruitment as well as following up with the enrolled patients [31]. The funding opportunities for new projects have also been severely hit across globe.

\section{Light at the End of Tunnel!}

The emergence of vaccines for COVID-19 is a boon, though the practicality of immunizing considerable population to achieve herd immunity is challenging in the Indian context. If everything goes according to estimates as given by the government, we may expect vaccination of at least 50-60\% of population by this year end. All individuals with active or prior cancer who are eligible for vaccination according to local allocation priorities should be advised by oncologists to get vaccinated. Although immunogenicity is attenuated, still vaccination is recommended in immunocompromised population. There is no reliable way to find whether vaccine has elicited protective immune response and CDC specifically recommend against the antibody testing to asses post vaccination immunity. Data are not currently available to establish vaccine safety and efficacy in patient with immunocompromised conditions such as cancer or immunosuppressive treatment.

\section{References}

1. Andrews MA, Areekal B, Rajesh KR et al (2020) First confirmed case of COVID-19 infection in India: a case report. Indian J Med Res 151:490-492

2. Murhekar MV, Bhatnagar T, Thangaraj JWV et al (2021) SARS$\mathrm{CoV}-2$ sero-prevalence among general population and healthcare workers in India, December 2020 - January 2021. Int J Infect Dis. https://doi.org/10.1016/j.ijid.2021.05

3. Liang W, Guan W, Chen R et al (2020) Cancer patients in SARSCoV-2 infection: a nationwide analysis in China. Lancet Oncol 21:335-337

4. Fillmore NR, La J, Szalat RE et al (2020) Prevalence and outcome of COVID-19 infection in cancer patients: a national veterans affairs study. J Natl Cancer Inst. https://doi.org/10.1093/jnci/ djaa159

5. Curigliano G, Banerjee S, Cervantes A et al (2020) Managing cancer patients during the COVID-19 pandemic: an ESMO multidisciplinary expert consensus. Ann Oncol 31:1320-1335
6. Hanson KE, Caliendo AM, Arias CA et al (2020) Infectious Diseases Society of America Guidelines on the Diagnosis of Coronavirus Disease 2019. Clin Infect Dis 16:760. https://doi. org/10.1093/cid/ciaa760

7. Patell R, Bogue T, Bindal P et al (2020) Incidence of thrombosis and hemorrhage in hospitalized cancer patients with Covid-19. J Thromb Haemost 18:23499-32357

8. Ueda M, Martins R, Hendrie PC et al (2020) Managing cancer care during the COVID-19 pandemic: agility and collaboration toward a common goal. J Natl Compr Canc Netw 20:1-4. https://doi.org/10.6004/jnccn.2020.7560

9. Curtis JR, Kross EK, Stapleton RD (2020) The importance of addressing advance care planning and decisions about do-notresuscitate orders during novel coronavirus 2019 (COVID-19). JAMA 323:1771-1772

10. Perni S, Milligan MG, Saraf A et al (2020) Treating the SARSCoV-2-positive patient with cancer: a proposal for a pragmatic and transparent ethical process. Cancer 126:3896-3899

11 Yekedüz E, Utkan G, Ürün Y (2020) A systematic review and meta-analysis: the effect of active cancer treatment on severity of COVID-19. Eur J Cancer 141:92-104

12 El-Boghdadly K, Cook TM, Goodacre T et al (2021) SARSCoV-2 infection, COVID-19 and timing of elective surgery. Anaesthesia 76:748-758

13. Avanzato VA, Matson MJ, Seifert SN et al (2020) Case study: prolonged infectious SARS-CoV-2 shedding from an asymptomatic immunocompromised individual with cancer. Cell 183:1901-1912

14. Desai S, Gupta A (2020) Indian J Surg Oncol 11:171-174

15. Cinar P, Kubal T, Freifeld A et al (2020) Safety at the time of the COVID-19 pandemic: how to keep our oncology patients and healthcare workers safe. J Natl Compr Canc Netw 18:504-558

16. https://www.mohfw.gov.in/pdf/Telemedicine.pdf. Accessed on 22 May 2021

17. Becker AS, Perez-Johnston R, Chikarmane SA et al (2021) Multidisciplinary recommendations regarding post-vaccine adenopathy and radiologic imaging Radiology Scientific Expert Panel. Radiology 24:210436. https://doi.org/10.1148/radiol.2021210436

18 Hanna TP, King WD, Thibodeau S et al (2020) Mortality due to cancer treatment delay: systematic review and meta-analysis. BMJ 371:m4087. https://doi.org/10.1136/bmj.m4087

19. Hartman HE, Sun Y, Devasia TP et al (2020) Integrated survival estimates for cancer treatment delay among adults with cancer during the COVID-19 pandemic. JAMA Oncol 6:1881-1889

20. COVID19 Subcommittee of the O.R. Executive Committee at Memorial Sloan Kettering (2020) Cancer Surgery and COVID19. Ann Surg Oncol 27:1713-1716. https://doi.org/10. 1245/s10434-020-08462-1

21. Thompson CK, Lee MK, Baker JL et al (2020) Taking a second look at neoadjuvant endocrine therapy for the treatment of early stage estrogen receptor positive breast cancer during the COVID19 outbreak. Ann Surg 272:e96-e97. https://doi.org/10.1097/SLA. 000000000000402

22. COVIDSurg Collaborative (2020) Mortality and pulmonary complications in patients undergoing surgery with perioperative SARS-CoV-2 infection: an international cohort study. Lancet 396:27-38

23. Cohn JA, Ghiraldi EM et al (2021) A critical appraisal of the American College of Surgeons Medically Necessary, Time Sensitive Procedures (MeNTS) scoring system, urology consensus recommendations and individual surgeon case prioritization for resumption of elective urological surgery during the COVID-19 pandemic. J urol 205:241-247

24. Kutikov A, Weinberg DS, Edelman MJ et al (2020) A war on two fronts: cancer care in the time of COVID-19. Ann Intern Med $172: 756-758$ 
25. Hanna TP, Evans GA, Booth CM (2020) Cancer, COVID-19 and the precautionary principle: prioritizing treatment during a global pandemic. Nat Rev Clin Oncol 17:268-270

26. Hwang ES, Balch CM, Balch GC et al (2020) Surgical oncologists and the COVID-19 pandemic: guiding cancer patients effectively through turbulence and change. Ann Surg Oncol 27:2600-2613

27. Berlin A, Lovas M, Truong T et al (2021) Implementation and outcomes of virtual care across a tertiary cancer centre during COVID-19. JAMA Oncol 7:597-602

28. Emanuel EJ, Persad G, Upshur R et al (2020) Fair allocation of scarce medical resources in the time of Covid-19. N Engl J Med 382:2049-2055

29. https://www.natboard.edu.in/viewNotice.php?NBE=T3NNN VRoc09xUW5kTHhLMXpJZFFRdz09. Accessed on 22 May 2021.
30. van Doesum J, Chinea A, Pagliaro M et al (2020) Clinical characteristics and outcome of SARS-CoV-2-infected patients with haematological diseases: a retrospective case study in four hospitals in Italy, Spain and the Netherlands. Leukemia 34:2536-2538

31. Araujo DV, Watson GA, Siu LL (2021) The day after COVID19-time to rethink oncology clinical research. JAMA Oncol 7:23-24. https://doi.org/10.1001/jamaoncol.2020.4240

Publisher's Note Springer Nature remains neutral with regard to jurisdictional claims in published maps and institutional affiliations. 